Published in final edited form as:

Sleep Med. 2019 May ; 57: 87-91. doi:10.1016/j.sleep.2019.01.030.

\title{
Characterizing Pediatric Inpatient Sleep Duration and Disruptions
}

\author{
Amarachi I. Erondu ${ }^{a}$, Nicola M. Orlov, MD ${ }^{a, b}$, Leah B. Peirce ${ }^{a}$, Samantha L. Anderson ${ }^{c}$, \\ Michael Chamberlain ${ }^{a}$, Kelsey Hopkins ${ }^{e}$, Christopher Lyttle, MA ${ }^{c}$, David Gozal, MD, MBA ${ }^{b, d}$, \\ Vineet M. Arora, MD, MAPPa,c \\ aPritzker School of Medicine, University of Chicago, Chicago, Illinois; \\ bSection of Academic Pediatrics, University of Chicago Medical Center, Chicago, Illinois; \\ 'Section of General Internal Medicine, University of Chicago Medical Center, Chicago, Illinois; \\ dSection of Pediatric Sleep Medicine, University of Chicago Medical Center, Chicago, Illinois; \\ eThe College of the University of Chicago, Chicago, Illinois
}

\begin{abstract}
Objective: To contextualize inpatient sleep duration and disruptions in a general pediatric hospital ward by comparing in-hospital and at-home sleep durations to recommended guidelines and objectively measure nighttime room entries.
\end{abstract}
Methods: Caregivers of patients 4 weeks - 18 years of age reported patient sleep duration and disruptions in anonymous surveys. Average at-home and in-hospital sleep durations were compared to National Sleep Foundation recommendations. Objective nighttime traffic was evaluated as the average number of room entries between 11:00pm and 7:00am using GOJO hand- hygiene room entry data.

Results: Among 246 patients, patients slept less in the hospital than at home with newborn and infant cohorts experiencing 7- and 4-hour sleep deficits respectively (Newborn: $787 \pm 318 \mathrm{~min}$ at home vs. $354 \pm 211 \mathrm{~min}$ in hospital, p<0.001; Infants: $530 \pm 115 \mathrm{~min}$ at home vs. $412 \pm 152 \mathrm{~min}$ in hospital, $\mathrm{p}<0.01$ ). Newborn children also experienced $>2$ hour sleep deficits at home when

\footnotetext{
Address Correspondence: Vineet Arora MD MAPP, Department of Medicine, University of Chicago Medicine, 5841 S Maryland Ave, MC 2007, Chicago IL 60637, [ varora@medicine.bsd.uchicago.edu].

Contributors' Statement Page

Amarachi Erondu aided in data collection, conducted the statistical analysis of the data, drafted the initial manuscript and reviewed and edited the final submission.

Leah Peirce and Michael Chamberlain aided in data collection and reviewed the final submission.

Kelsey Hopkins aided in data collection, statistical analysis, and reviewed the final submission.

Samantha Anderson developed the databases for data collection, aided in survey administration and reviewed the final submission. Christopher Lyttle aided in the statistical analysis of the data and reviewed the final submission.

Dr. David Gozal conceptualized and designed the study and reviewed the final submission.

Dr. Nicola Orlov and Dr. Vineet Arora conceptualized and designed the study, conducted the statistical analysis of the data, aided in drafting the initial manuscript and reviewed and edited the final submission.

Publisher's Disclaimer: This is a PDF file of an unedited manuscript that has been accepted for publication. As a service to our customers we are providing this early version of the manuscript. The manuscript will undergo copyediting, typesetting, and review of the resulting proof before it is published in its final citable form. Please note that during the production process errors may be discovered which could affect the content, and all legal disclaimers that apply to the journal pertain.

Conflict of Interest: None
} 
compared to NSF recommendations (Newborns: $787 \pm 318 \mathrm{~min}$ at home vs. $930 \mathrm{~min}$ recommended, $\mathrm{p}<0.05$ ). Objective nighttime traffic measures revealed that hospitalized children experienced 10 room entries/night ( $10 \pm 5.9$ entries). Nighttime traffic was significantly correlated with caregiver-reported nighttime awakenings (Spearman Rank Correlation Coefficient: 0.58, p=. 04).

Conclusion: Hospitalization is a missed opportunity to improve sleep both in the hospital and at home.

\section{Keywords}

sleep; pediatrics; hospital

\section{Introduction}

Adequate sleep plays a crucial role in the optimal growth and development of children and adolescents ${ }^{1}$. Studies have also shown that getting an appropriate amount of sleep as a child is associated with a decreased risk of developing chronic conditions such as obesity or Type II diabetes later in life $\mathrm{e}^{2,3}$. The numerous benefits afforded by adequate sleep have prompted organizations like the National Sleep Foundation (NSF) to release recommended ranges for sleep durations for newborns, infants, toddlers, school-aged children, teenagers, and young adults $^{4}$ (Appendix 1). Sufficient sleep is particularly important for ill children as it can facilitate the recovery process by promoting protein synthesis, cell-division and tissue repair mechanisms 5 .

Unfortunately, in spite of the many benefits of adequate sleep for growth, development, and recovery from illness, periods of hospitalization are often associated with complaints of poor sleep quality among pediatric inpatients ${ }^{5,6}$. Studies of sleep quality among hospitalized children could provide useful information needed to develop interventions that improve sleep in the hospital inpatient setting. However, in order to develop targeted interventions, researchers must recognize that sleep in the hospital does not occur within a vacuum and is, instead, influenced by the hospital environment and pre-established sleep hygiene behaviors that affect baseline sleep. Though many studies investigating hospitalized sleep in children admitted to general pediatric wards compare home and hospital sleep durations, few compare sleep durations in the hospital and at home to recommended sleep guidelines to assess whether sleep deficits are occurring at baseline. Further, few studies utilize objective measures of sleep disruptions to assess the true impact of the hospital environment on patient sleep $5,7,8,9$.

We hypothesized that the overall duration of sleep would be reduced in the inpatient setting among hospitalized children and that sleep disruptions in the form of nighttime room entries are frequent within the hospital setting. Therefore, we here aimed to characterize inpatient pediatric sleep by comparing subjective measures of home and hospital sleep durations to recommended pediatric sleep guidelines and to quantify hospitalized sleep disruptions using objective measures. 


\section{Methods}

\subsection{Study Population}

This study was reviewed and approved by the Institutional Review Board of the University of Chicago Medicine and verbal consent was obtained from all study participants. A convenience sample of patients was recruited in the general pediatric inpatient ward of Comer Children's Hospital. Eligible patients included all children between the ages of 4 weeks and 18 years admitted to the ward which also includes the neurology or gastroenterology inpatient specialty services. All the caregivers approached in this study had spent the night in the hospital room with their child and all the patients included in the study slept in single rooms. Children recovering from recent surgeries are not admitted to the general pediatric ward and, therefore, were not included in the study. Further exclusions were made to identify a study population that represents a general population of healthy children without known chronic diseases that may impact sleep at baseline. Therefore, we excluded patients with established diagnoses or recent medical procedures that are known disruptors of sleep. This included patients with a known diagnosis of cerebral palsy, tracheostomy, nasogastric or gastrostomy tube dependence, sleep apnea or altered mental status. Patients were also excluded if they were on bed rest or were undergoing long term monitoring for epilepsy.

\subsection{Data Collection}

2.2.1 Survey Instruments-Caregivers were asked to complete a Pediatric Sleep Assessment upon recruitment. This survey contained several validated instruments including the Karolinska Sleep Log and the Pediatric Sleep Questionnaire (PSQ) ${ }^{15}$. The PSQ is a tool that screens for pediatric sleep-disordered breathing (SDB) in children 2-18 years of age based on the presence of symptoms such as snoring, daytime sleepiness, and behavioral problems and was used in this study to further characterize our study population ${ }^{16}$. Data from survey responses were stored using the REDCap Database Version 6.14.0 (Vanderbilt University, 2016, Nashville, TN) ${ }^{17}$.

\subsubsection{Caregiver-Reported Sleep Duration \& Disruptions-Caregivers reported} patient sleep duration and sleep disruptions at home and in the hospital by completing the Karolinska Sleep Log. This tool has been validated against EEG variables and includes questions regarding the total number of hours a patient slept and the total number of awakenings experienced during nighttime sleep ${ }^{15}$. Recruited caregivers were asked to provide these values for a typical night of sleep at home within the past month when their child was not ill and for the previous night of sleep within the hospital. Caregivers were also asked to report daytime sleep duration within this survey.

\subsubsection{Objectively Measured Sleep Disruptions: Nighttime traffic-Objective} data on nighttime traffic in and out of patient rooms were collected using data from Activity Counters provided by the GOJO SMARTLINK Hand-Hygiene system (GOJO Industries Inc., 2017, Akron, OH). These activity counters employ heat sensors to track all patient room entries and exits. This information is used by the hospital as a denominator for 
potential hand hygiene opportunities. For the purpose of this study, data from these devices were obtained from the hospital to measure nighttime room entries (Supplemental Figure 1).

\subsection{Data Analysis}

Descriptive statistics were used to summarize data. Subjective data were separated into newborn, infant, toddler, preschooler, school-aged, teenager and young adult cohorts to mirror National Sleep Foundation recommendations ${ }^{4}$. The average durations for sleep in the hospital and at home within the two age groups were compared using paired t-tests. In addition, the observed values for sleep duration in the hospital and at home for each cohort were compared to age-appropriate sleep guidelines set by the National Sleep Foundation using t-tests.

\subsubsection{Objectively Measured Sleep Disruptions: Nighttime Traffic-To analyze} our objective room entry data, we first defined "nighttime traffic" as all room-exits occurring between 11:00pm and 7:00am. We chose this definition to exclude nighttime room entries by patient family members that were not paired with a subsequent exit. A single room entry was defined as the total number of room exits that occurred within a three-minute window ${ }^{18}$. The average number of room entries within a single night was then calculated.

Caregiver responses for the number of nighttime hospital sleep awakenings (collected from the Karolinska Sleep Log) were averaged by month and variation was compared to monthly changes in objectively measured nighttime room entries by calculating the Spearman rank correlation coefficient. All data were analyzed using STATA 15.019. Statistical significance was set as a p-value $<0.05$.

\section{Results}

\subsection{Study Population}

From February 2017 to April 2018, a total of 246 patients were recruited and completed the Pediatric Sleep Assessment. Of the recruited patients, $42 \%$ were female and 59\% were older than 2 years (Table 1). Almost half of the participating children were the youngest children in their family (48\%), and the majority of the caregivers surveyed in this study were mothers (77\%). Patients recruited for this study were also diverse in racial and ethnic backgrounds with $72 \%$ of respondents identifying as African American, and 10\% of respondents identifying as Hispanic. The most common admitting diagnoses in our study population were upper or lower respiratory tract infections (21\%), asthma exacerbations (14\%), acute conditions pertaining to the GI tract (7\%) and skin and soft tissue infections (6\%). Use of the Pediatric Sleep Questionnaire revealed that $24 \%$ of patients $\geq 2$ years screened positive for high risk of sleep-disordered breathing.

\subsection{Caregiver-Reported Subjective Sleep Duration}

On average, patients slept less in the hospital than at home with the newborn and infant cohort experiencing 7- and 4-hour sleep deficits respectively (Newborn: $787 \pm 318 \mathrm{~min}$ at home vs. $354 \pm 211 \mathrm{~min}$ in hospital, p<0.001; Infants: $530 \pm 115 \mathrm{~min}$ at home vs. $412 \pm 152$ min in hospital, $\mathrm{p}<0.01$ ). Additionally, study participants had at-home and in-hospital sleep 
durations that were below the age-related NSF recommendations ${ }^{4}$ (Figure 1) with newborn and infant children experiencing the greatest deficits. For infant children, the average reported sleep duration at home fell shy of recommendations by approximately 1.5 hours (Infants: $703 \pm 203 \mathrm{~min}$ at home vs. $810 \mathrm{~min}$ recommended, $\mathrm{p}=0.09$ ). Newborn children exhibited an average sleep debt of $>2$ hours when comparing at-home sleep duration to recommended sleep guidelines (Newborns: $787 \pm 318 \mathrm{~min}$ at home vs. $930 \mathrm{~min}$ recommended, $\mathrm{p}<0.05)$. For the whole cohort, only $23 \%$ of caregivers reported at home sleep durations that met NSF standards.

\subsection{Objectively Measured Sleep Disruptions: Nighttime Traffic}

Hospitalized children with inpatient stays between February 2017 and April 2018 experienced an average of 10 room entries within a single night in the hospital $(10 \pm 5.9$ nighttime room entries). Further, monthly averages of objectively measured nighttime room entries (between February 2017 and February 2018) were significantly correlated with monthly variation in the average number of caregiver-reported nighttime awakenings (Spearman Rank Correlation Coefficient: 0.58, p=.04) (Figure 2).

\section{Discussion}

In this study, we found that, regardless of age, caregivers reported less sleep in the hospital when compared to the duration of sleep reported for their children at home. These results are consistent with prior studies that focus on patients admitted to general pediatric units, and suggest that acutely ill pediatric inpatients are at increased risk to experience sleep deficits while in the hospital ${ }^{5,7,8}$. Further, most of the caregivers in this study reported sleep durations for their children in the hospital and at home that were below the recommended guidelines set by the National Sleep Foundation ${ }^{4}$ with newborn patients experiencing the greatest deficits, suggesting that sleep deficits are pervasively present among newborn children independent of their health status.

Previous studies have suggested that poor sleep in the week prior to hospital admission was the strongest predictor of poor sleep within the hospital, prompting the assumption that the poor sleep observed during hospitalization could be related to the pathology causing the study participant's admission to the hospital ${ }^{7}$. Though our study also demonstrated sleep deficits at home and in the hospital, the assessment of typical home sleep duration, presumably when the child is not acutely ill, suggests that the sleep deficits observed at home could also be the result of chronic poor sleep hygiene, rather than be ascribable to the acute illness that prompted their admission. These findings illustrate that within this predominantly minority urban patient population, periods of hospitalization may be missed opportunities for patient and caregiver education surrounding overall sleep habits, and that educational efforts addressing sleep may need to be more holistic in nature, and include initiatives that reinforce the importance of sleep at home.

This study is the first to incorporate objective measures of hospital sleep disruptions to characterize pediatric inpatient sleep $5,7,8,20$. We found that pediatric patients hospitalized between February 2017 and April 2018 experienced an average of 10 nighttime room entries. These results provide a better insight to sleep in the hospital by objectively 
illustrating the magnitude of sleep disruptors that can be attributed to the hospital environment. The fact that the monthly variation recorded by the sensors is significantly correlated with monthly changes in parallel caregiver reports of nighttime awakenings suggests that hospital-based factors are likely impacting the ability of hospitalized children to achieve optimal sleep during their inpatient stays. This finding also aligns with prior studies that have exclusively used subjective caregiver reports to illustrate that nurse or physician interruptions lead to increased nighttime awakenings for hospitalized children ${ }^{5,7,8}$. Thus, there is a clear need for targeted interventions that decrease nighttime sleep interruptions in hospital settings. One example of such an intervention would involve changing the electronic medical record order sets, such that medication administration and blood draws are conducted at times that are not disruptive to the patient's sleep. Other options could allow nurses to forego overnight vital sign checks on patients when they are not critically indicated, ultimately resulting in anticipated decreases in the number of sleep interruptions that these patients will experience.

We also found that there was seasonal variation in the number of nighttime room entries observed with the number of room entries nearly doubling in the winter months. This is most likely due to the increased number of acutely ill children admitted to the hospital with diagnoses such as influenza or upper respiratory infections that may require increased monitoring by nursing staff.

Interestingly, we found that $24 \%$ of our patients screened positive for high-risk of sleepdisordered breathing- a figure that is markedly higher than the reported prevalence in the general population ${ }^{10,11}$. Though we did not use polysomnography to confirm the putative SDB diagnosis, the Pediatric Sleep Questionnaire's positive predictive value suggests that $\sim 85 \%$ of the hospitalized patients screening positive for high-risk of SDB would de facto be confirmed as suffering from SDB via polysomnography. This high prevalence in hospitalized patients may be explained by the fact that SDB often presents with conditions commonly seen in acutely ill hospitalized children such as severe asthma or lower respiratory tract infections ${ }^{12,13,14}$ The high prevalence could also be explained by studies that have clearly documented that patients with sleep disorders such as sleep apnea are highutilizers of healthcare services with elevated rates of emergency room visits and hospital admissions $^{13}$

An important limitation of our analysis of hospitalized sleep duration is the exclusive reliance on subjective reports from caregivers which may be susceptible to recall bias. In future studies, we plan on utilizing objective measures of sleep duration and quality such as actigraphy to address this potential issue. In addition, this study was conducted at a single institution with an underserved patient population, thus, limiting its generalizability. Another limitation of the study is the fact that we did not assess whether our objective room entry data correlated with caregiver responses for nighttime awakenings at the room level. The absence of this analysis can be explained by the fact that we did not include protected health information (e.g. patient room numbers) in our survey data. In addition, our use of the Karolinska Sleep Log is another limitation as this instrument has not been validated in pediatric populations. Finally, the lack of data on daytime sleep and parent sleep at home 
and in the hospital further limits the ability to develop a more holistic understanding of sleep debt incurred within the hospital.

\section{Conclusions}

Notwithstanding the recognized fact that sufficient sleep is crucial for overall health and wellness, development, and recovery from illness, hospitalized children and their families report sleep durations and disruptions indicative of poor sleep quality, both in the hospital and at home, but particularly in the hospital. The large differences between recommended sleep duration and the sleep duration reported in the hospital and at home suggest that the hospital sleep deficits observed in newborn and infant patients could be related, at least in part, to underlying poor sleep hygiene at baseline. Further, our objective analysis of nighttime traffic in the patient rooms suggests that the fewer hours of sleep that children attain in the hospital are plagued with frequent sleep interruptions-often in the form of numerous room entries over the course of the night.

This study adds to the extant literature by suggesting that poor sleep quality in a pediatric inpatient population may be due to pre-admission poor sleep hygiene behaviors, a disruptive hospital environment, and/or underlying clinical diagnoses negatively impacting sleep. Our study also highlights that periods of hospitalization constitute missed opportunities to address some of these issues through more targeted interventions that improve education of caregivers around proper sleep hygiene for their children and initiatives that promote better sleep in hospital environments.

\section{Supplementary Material}

Refer to Web version on PubMed Central for supplementary material.

\section{Acknowledgements:}

We would like to acknowledge funding by the National Heart, Lung, and Blood Institute [R25HL0963283-08]; NHLBI [5R25HL116372-02]; and NHLBI [1K24HL136859]. We would also like to thank Jillian Bowman for her assistance in data collection.

Funding Source: This work was funded by the National Heart, Lung, and Blood Institute [R25HL0963283-08]; NHLBI [5R25HL116372-02]; and NHLBI [1K24HL136859].

\section{Appendix:}

Table 2:

National Sleep Foundation Recommendations for Sleep Duration for Children

\begin{tabular}{|c|c|}
\hline & Recommended Sleep Duration \\
\hline Newborns (0-3 Months) & $14-17 \mathrm{hrs}$ \\
\hline Infants (4-11 Months) & $12-15 \mathrm{hrs}$ \\
\hline Toddlers (1-2 Years) & $11-14 \mathrm{hrs}$ \\
\hline Preschoolers (3-5 Years) & $10-13 \mathrm{hrs}$ \\
\hline School-Aged Children (6-13 Years) & $9-11 \mathrm{hrs}$ \\
\hline
\end{tabular}




\begin{tabular}{|c|c|}
\hline & Recommended Sleep Duration \\
\hline Teenagers (14-17 Years) & $8-10 \mathrm{hrs}$ \\
\hline Young Adults (18-21 Years) & $7-9 \mathrm{hrs}$ \\
\hline
\end{tabular}

\section{References}

1. Eastman CJ, Lazarus L. Growth hormone release during sleep in growth retarded children. Arch Dis Child. 1973;48(7):502-507. [PubMed: 4719103]

2. Anderson SE, Andridge R, Whitaker RC. Bedtime in Preschool-Aged Children and Risk for Adolescent Obesity. J Pediatr. 2016;176:17-22. doi:10.1016/j.jpeds.2016.06.005 [PubMed: 27426836]

3. Rudnicka AR, Nightingale CM, Donin AS, et al. Sleep Duration and Risk of Type 2 Diabetes. Pediatrics. 2017;140(3):e20170338. doi:10.1542/peds.2017-0338 [PubMed: 28811317]

4. Hirshkowitz M, Whiton K, Albert SM, et al. National Sleep Foundation's sleep time duration recommendations: methodology and results summary. Sleep Health: Journal of the National Sleep Foundation. 2015;1(1):40-43. doi:10.1016/j.sleh.2014.12.010

5. Meltzer LJ, Davis KF, Mindell JA. Patient and parent sleep in a children's hospital. Pediatr Nurs. 2012;38(2):64-71; [PubMed: 22685865]

6. Coyne I Children's experiences of hospitalization. J Child Health Care. 2006;10(4):326-336. doi: 10.1177/1367493506067884 [PubMed: 17101624]

7. Herbert AR, de Lima J, Fitzgerald DA, Seton C, Waters KA, Collins JJExploratory study of sleeping patterns in children admitted to hospital. J Paediatr Child Health. 2014;50(8):632-638. doi:10.1111/ jpc.12617 [PubMed: 24893889]

8. Bisogni S, Chiarini I, Giusti F, Ciofi D, Poggi GM, Festini F. Impact of hospitalization on the sleep patterns of newborns, infants and toddlers admitted to a pediatric ward: a cross-sectional study. Minerva Pediatr. 2015;67(3):209-217. [PubMed: 25297322]

9. Stremler R, Adams S, Dryden-Palmer K. Nurses' views of factors affecting sleep for hospitalized children and their families: A focus group study. Res Nurs Health. 2015;38(4):311-322. doi: 10.1002/nur.21664 [PubMed: 25970699]

10. Rosen CL. Clinical features of obstructive sleep apnea hypoventilation syndrome in otherwise healthy children. Pediatr Pulmonol. 1999;27(6):403-409. [PubMed: 10380092]

11. Bixler EO, Vgontzas AN, Lin H-M, et al. Sleep Disordered Breathing in Children in a General Population Sample: Prevalence and Risk Factors. Sleep. 2009;32(6):731-736. [PubMed: 19544748]

12. Ross KR, Storfer-Isser A, Hart MA, et al. Sleep-Disordered Breathing is Associated with Asthma Severity in Children. The Journal of Pediatrics. 2012;160(5):736-742. doi:10.1016/j.jpeds. 2011.10.008 [PubMed: 22133422]

13. Tarasiuk A, Greenberg-Dotan S, Simon-Tuval T, et al. Elevated morbidity and health care use in children with obstructive sleep apnea syndrome. Am J Respir Crit Care Med. 2007;175(1):55-61. doi:10.1164/rccm.200604-577OC [PubMed: 17038661]

14. Kheirandish-Gozal L, Dayyat EA, Eid NS, Morton RL, Gozal D. Obstructive sleep apnea in poorly controlled asthmatic children: effect of adenotonsillectomy. Pediatr Pulmonol. 2011 Sep;46(9): 913-8. doi: 10.1002/ppul.21451.

15. Keklund G, Akerstedt T. Objective components of individual differences in subjective sleep quality. J Sleep Res. 1997;6(4):217-220. [PubMed: 9493520]

16. Chervin RD, Hedger K, Dillon JE, Pituch KJ. Pediatric sleep questionnaire (PSQ): validity and reliability of scales for sleep-disordered breathing, snoring, sleepiness, and behavioral problems. Sleep Medicine. 2000;1(1):21-32. doi:10.1016/S1389-9457(99)00009-X [PubMed: 10733617]

17. Harris Paul A., Taylor Robert, Thielke Robert, Payne Jonathon, Gonzalez Nathaniel, Conde Jose G., Research electronic data capture (REDCap) - A metadata-driven methodology and workflow 
process for providing translational research informatics support, J Biomed Inform. 2009 4;42(2): 377-81. [PubMed: 18929686]

18. Burke R, Auerbach A. Web Exclusives. Annals for Hospitalists Inpatient Notes - Research Highlights From Hospital Medicine 2017. Ann Intern Med. 2017;166(12):HO2-HO3. doi: 10.7326/M17-1243

19. StataCorp. 2017 Stata Statistical Software: Release 15. College Station, TX: StataCorp LLC

20. Oliveira L, Gomes C, Bacelar Nicolau L, Ferreira L, Ferreira R. Environment in pediatric wards: light, sound, and temperature. Sleep Med. 2015;16(9):1041-1048. doi:10.1016/j.sleep.2015.03.015 [PubMed: 26298777] 


\section{Highlights:}

1. Across all ages, pediatric patients slept less in the hospital than at home

2. Newborns slept over 2 hrs less than National Sleep Foundation guidelines at home

3. Study participants experienced an average of 10 hospital room entries each night

4. Nighttime traffic significantly correlated with reported nighttime awakenings 


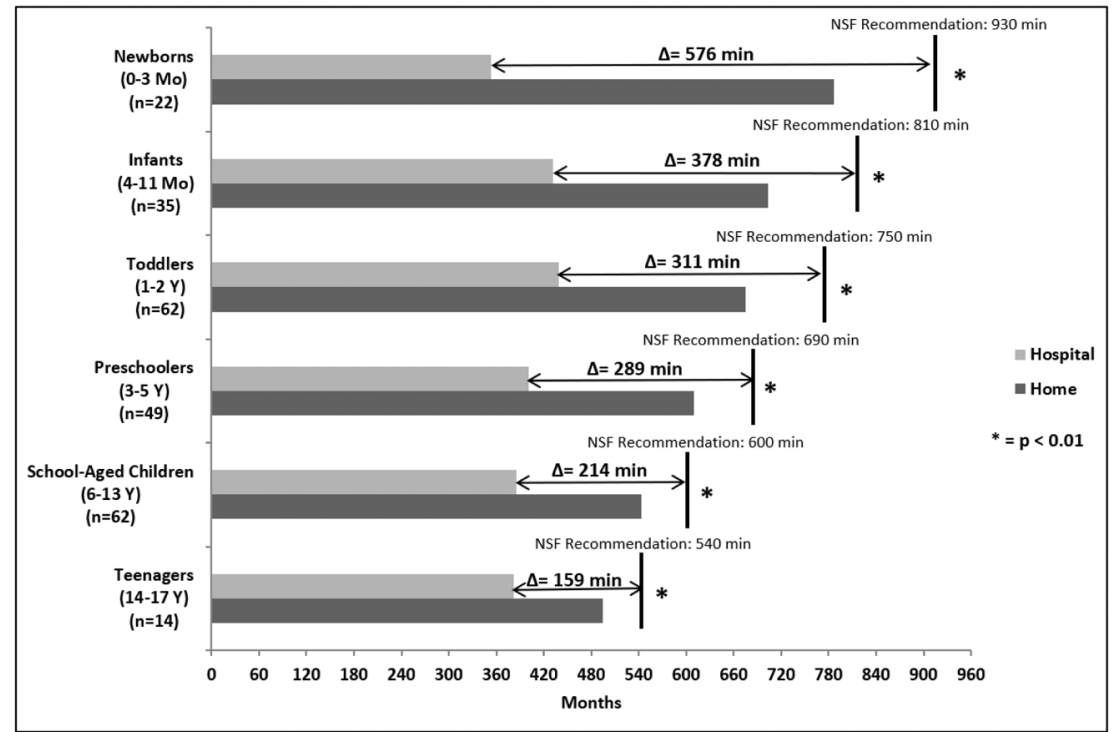

Figure 1: Comparing at home and in-hospital sleep duration to recommended pediatric patient guidelines for nighttime sleep

Sleep duration was reported by caregivers using the Karolinska Sleep Log within a Pediatric Sleep Assessment ${ }^{15}$. Recommended sleep duration values for children are averages of agespecific ranges published by the National Sleep Foundation (NSF) ${ }^{4}$. 


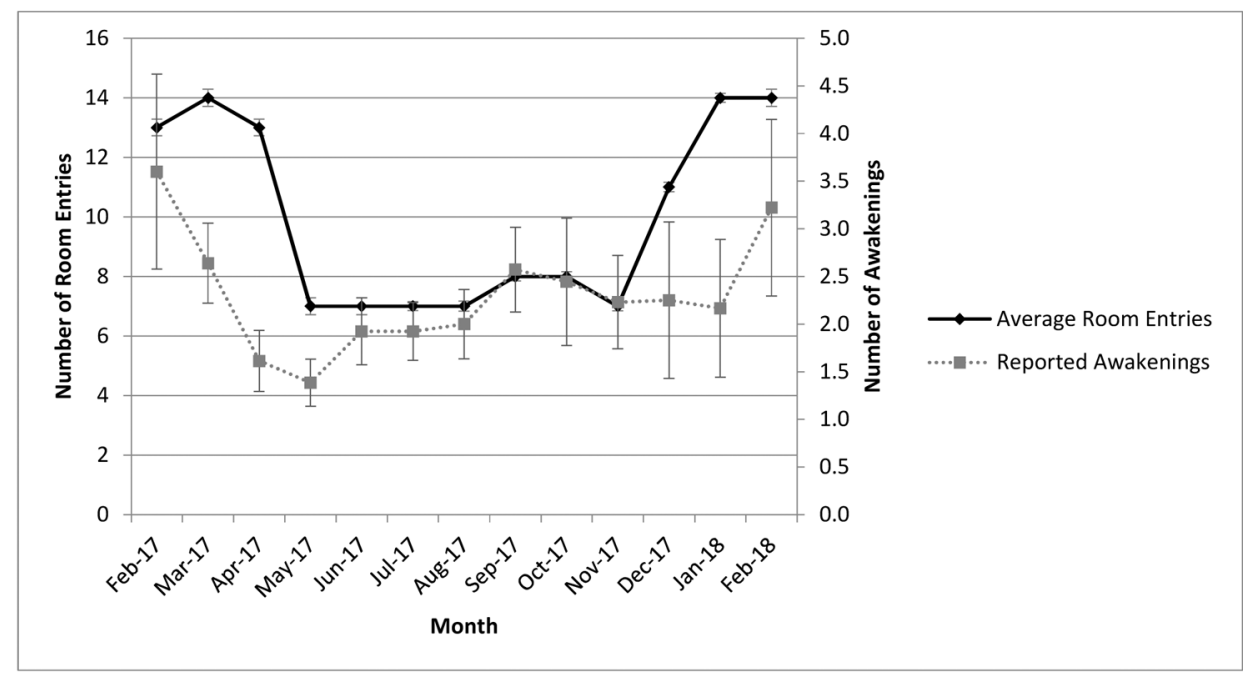

Figure 2: Monthly average of objectively measured nighttime room entries and number of caregiver-reported of nighttime awakenings in the hospital

Data from activity counters within hand-hygiene heat sensors inside of each patient room in the general pediatric ward were obtained to objectively measure nighttime room entries. Caregiver reports regarding number of nighttime awakenings were drawn from responses to the Karolinska Sleep $\log ^{15}$. Monthly variation in the average number of objectively measured nighttime room entries varied in a similar manner as monthly changes in the average number of caregiver-reported nighttime awakenings within the hospital (Spearman Rank Correlation Coefficient: 0.58, $\mathrm{p}=.04$ ). 
Table 1:

Study Population Demographics

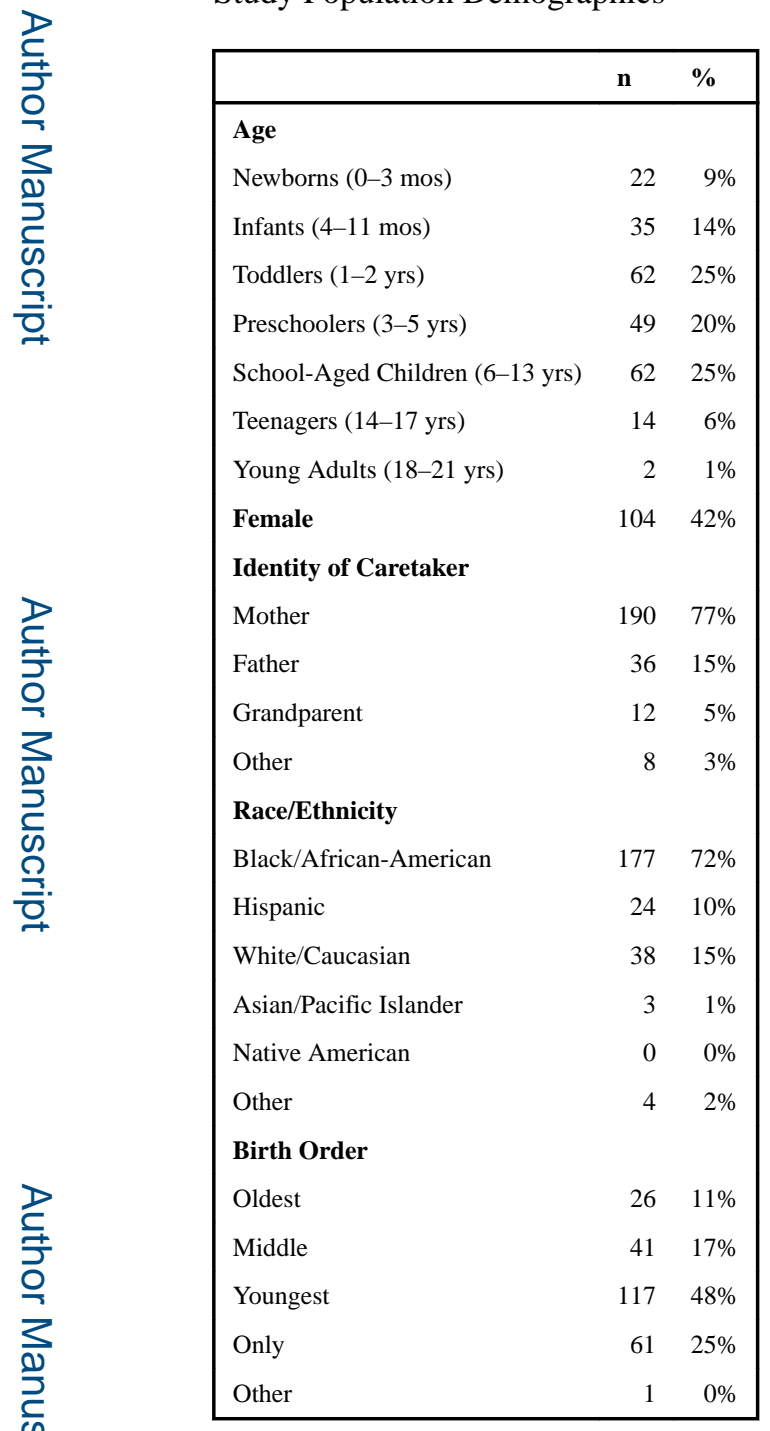

A convenience sample of patients on a general pediatric inpatient floor of Comer Children's Hospital in Chicago, Illinois, was recruited for surveys pertaining to sleep at home and within the hospital. 